\title{
Signal-based diagnostics of the gasoline injection engine
}

\author{
Marius Singureanu * and Ion Copae \\ Department of Military Vehicles and Transportation, Military Technical Academy "Ferdinand I", 39-49 George Cosbuc Blvd., \\ Bucharest.
}

Global Journal of Engineering and Technology Advances, 2021, 09(02), 083-091

Publication history: Received on 18 October 2021; revised on 22 November 2021; accepted on 24 November 2021

Article DOI: https://doi.org/10.30574/gjeta.2021.9.2.0156

\begin{abstract}
The paper addresses one of the methods of diagnosing the gasoline injection engine available on the vehicle, namely signal-based diagnostics. The most used algorithms for signal-based diagnostics are highlighted. The possibilities offered on an experimental basis are presented and the tests performed in this respect are presented. Time-frequency analysis techniques are applied to detect defects caused in the case of the Audi A6 car engine.
\end{abstract}

Keywords: Signal-based diagnosis; Fault; Failure; Vehicle engine; Time-frequency analysis

\section{Introduction}

In the case of signal-based diagnosis, it is necessary to compare the measured quantity in the event of a fault with the corresponding quantity in the case of normal operation; thus detecting the deviation of the target variable from a normal engine operating mode. For this purpose, the following are used for quantitative or qualitative assessments: first-order statistical characteristics (mean value, variance, standard deviation, entropy, and so on), monospectral (Fourier transform) or bispectral (higher order cumulants), frequency analysis, time-frequency analysis (spectrogram, Cohen class transforms, Stockwell transform, wavelet transform, and so on), cyclostationary analysis (frequency-frequency), probabilistic methods (Bayesian techniques / Bayesian decisions), detection of sudden / abrupt variations etc. As can be seen, these methods do not require mathematical models of gasoline engine operation (as in model-based diagnostics), but involve the existence of constructive elements that provide values of those variables and this aspect shows the increased role of sensors and actuators in engine diagnostics [2, 3, 6, 7]. As noted, signal-based analysis applies to time domain, frequency domain, time-frequency domain, and frequency-frequency domain. The paper will only use time analysis and time-frequency analysis to diagnose the engine.

\section{Experimental research on engine operation without faults}

As mentioned, for signal-based diagnosis, functional variables must be compared in the event of a fault and in the faultfree situation. In this sense, experimental research was carried out with an Audi A6 3.0 TFSI Quattro car equipped with a gasoline injection engine (fig.1) and functional variables were recorded using the Ross-Tech VCDS tester and specialized software for the VAG group. During the experiments, the functional variables were measured for 30 faultfree tests, denoted A1-A30. With the help of these tests, mathematical models of engine operation without faults were established, which allowed the comparison with the cases in which faults were caused.

For example, Fig. 2 shows the instantaneous values of the $M_{e}$ engine torque, where the extreme values are also shown, as well as the range $40-150 \mathrm{Nm}$ with the most values $(69.2 \%)$.

\footnotetext{
${ }^{*}$ Corresponding author: Ion Copae

Department of Military Vehicles and Transportation, Military Technical Academy "Ferdinand I", 39-49 George Cosbuc Blvd., Bucharest.
} 


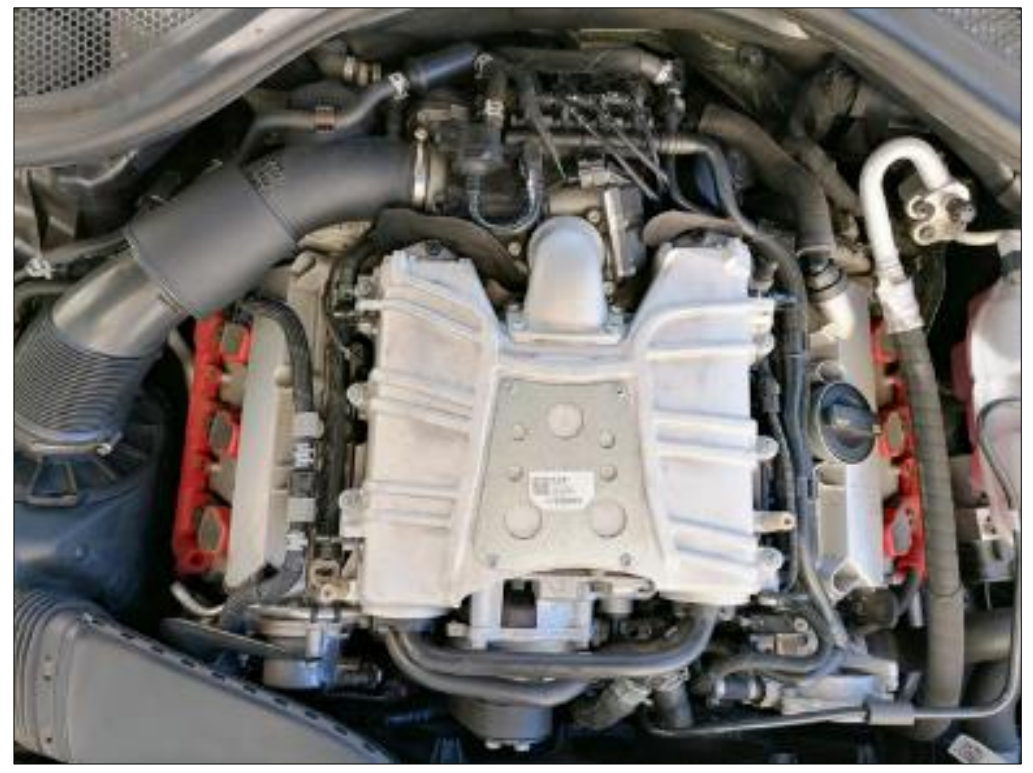

Figure 1 Audi A6 3.0 TFSI Quattro car engine

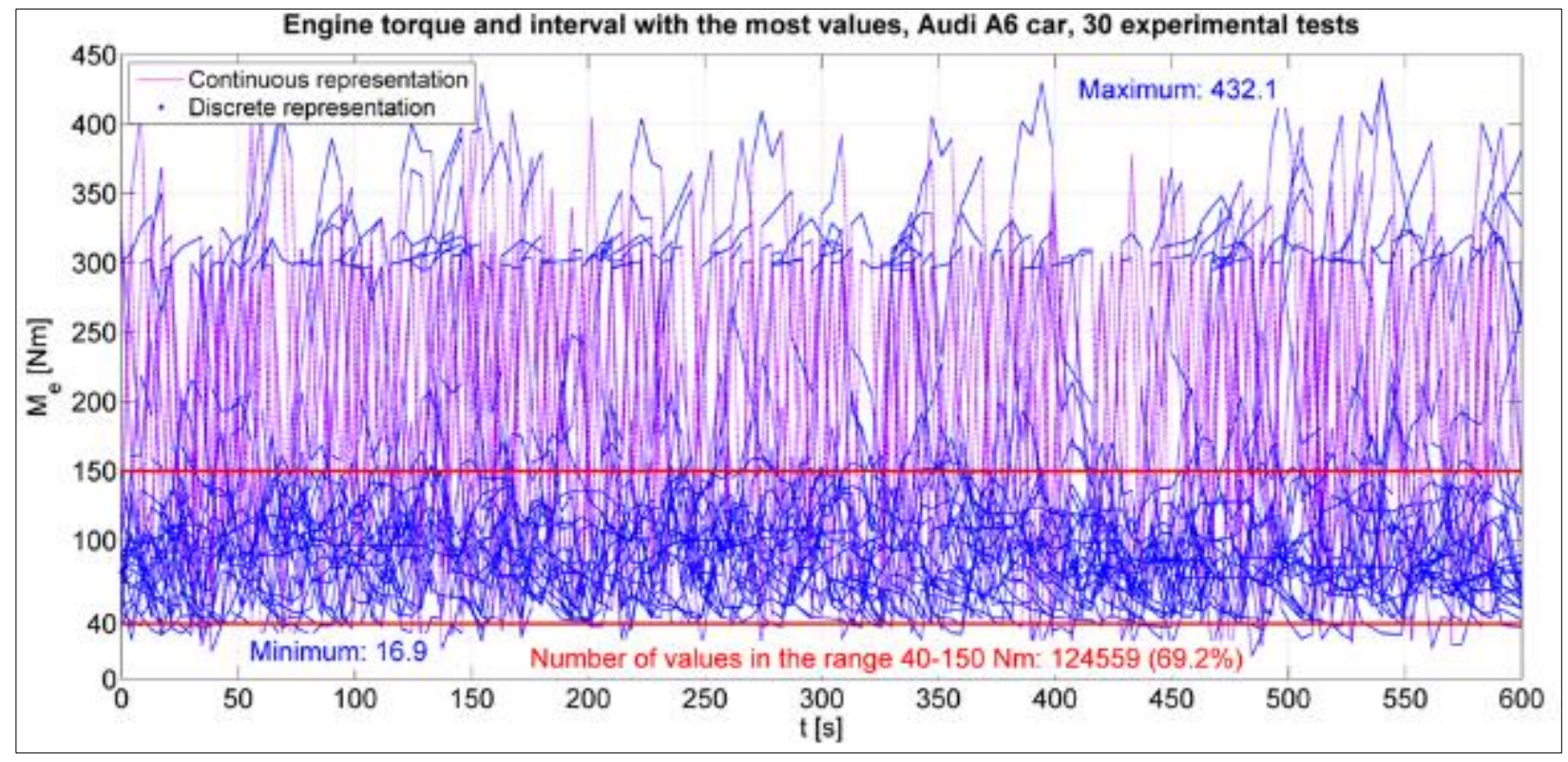

Figure 2 Instantaneous torque values, 30 tests, Audi A6 car

Fig. 3 shows the maximum values on the tests of the engine torque $M_{e}$, the engine power $P_{e}$, the fuel pressure $p_{c}$ and the intake air pressure $p_{a}$. The graphs also show the maximum values of the torque and power of the engine from its technical specifications; also, the extreme and average values for all 30 samples are shown.

Fig. 4 shows the average values on tests of the hourly fuel consumption $C_{h}$, the cyclic flow of fuel $c_{c}$, the effective specific consumption $c_{e}$ and the consumption per $100 \mathrm{~km} C_{100}$. The graphs also show the extreme and average values for all 30 tests.

Fig. 5 shows the result of establishing the spatial static characteristic $\left.M_{e}=\mathrm{f}(n, \xi)\right)$ of the engine of the Audi A6 car. The graph shows the switching surface of the static characteristic, its analytical expression, as well as the values of the 30 experimental tests.

Similarly, Fig. 6 shows the spatial static characteristic $\left.C_{a}=\mathrm{f}(n, \xi)\right)$ of the air supply system, where the hourly air consumption $C_{a}$ is measured. 


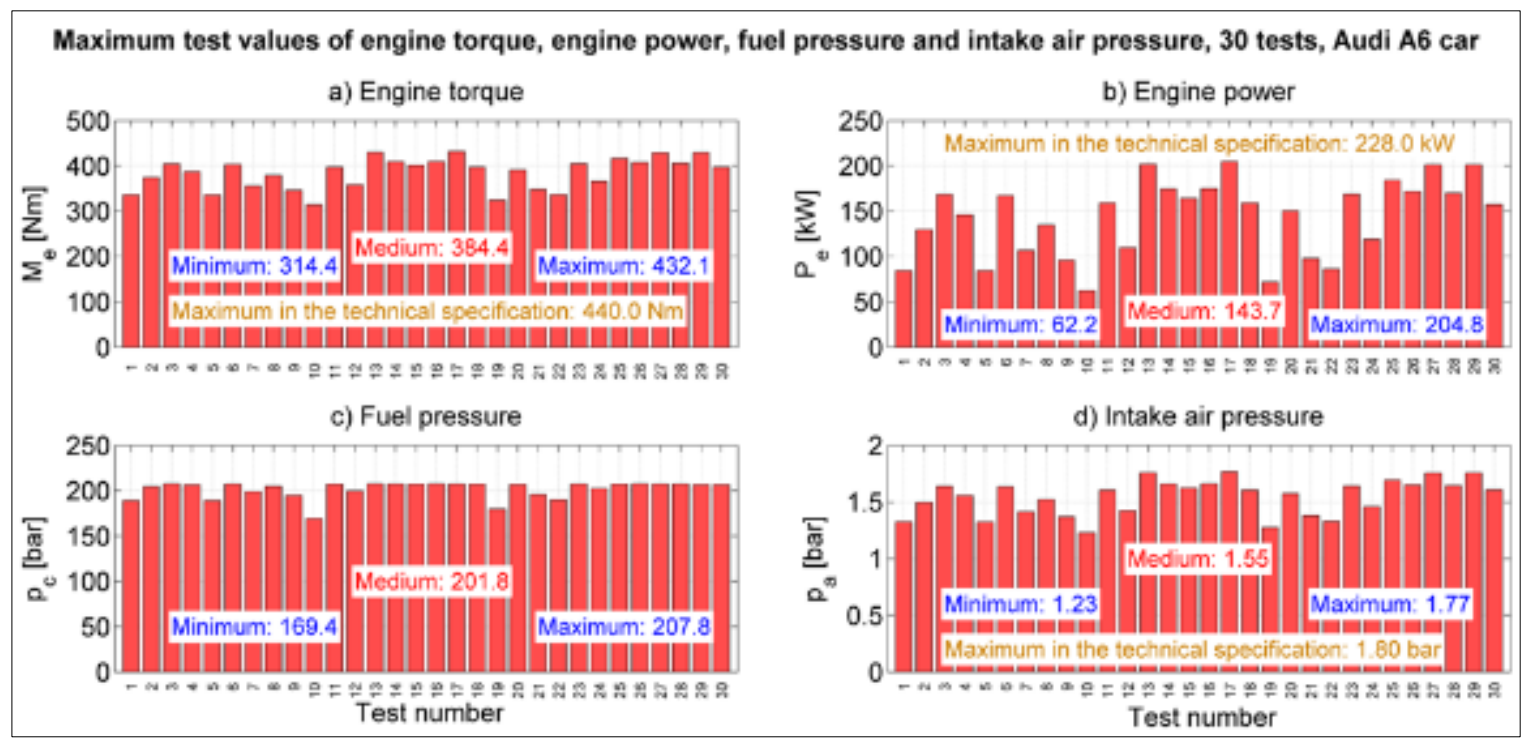

Figure 3 Maximum values per test for four functional variables, 30 tests, Audi A6 car

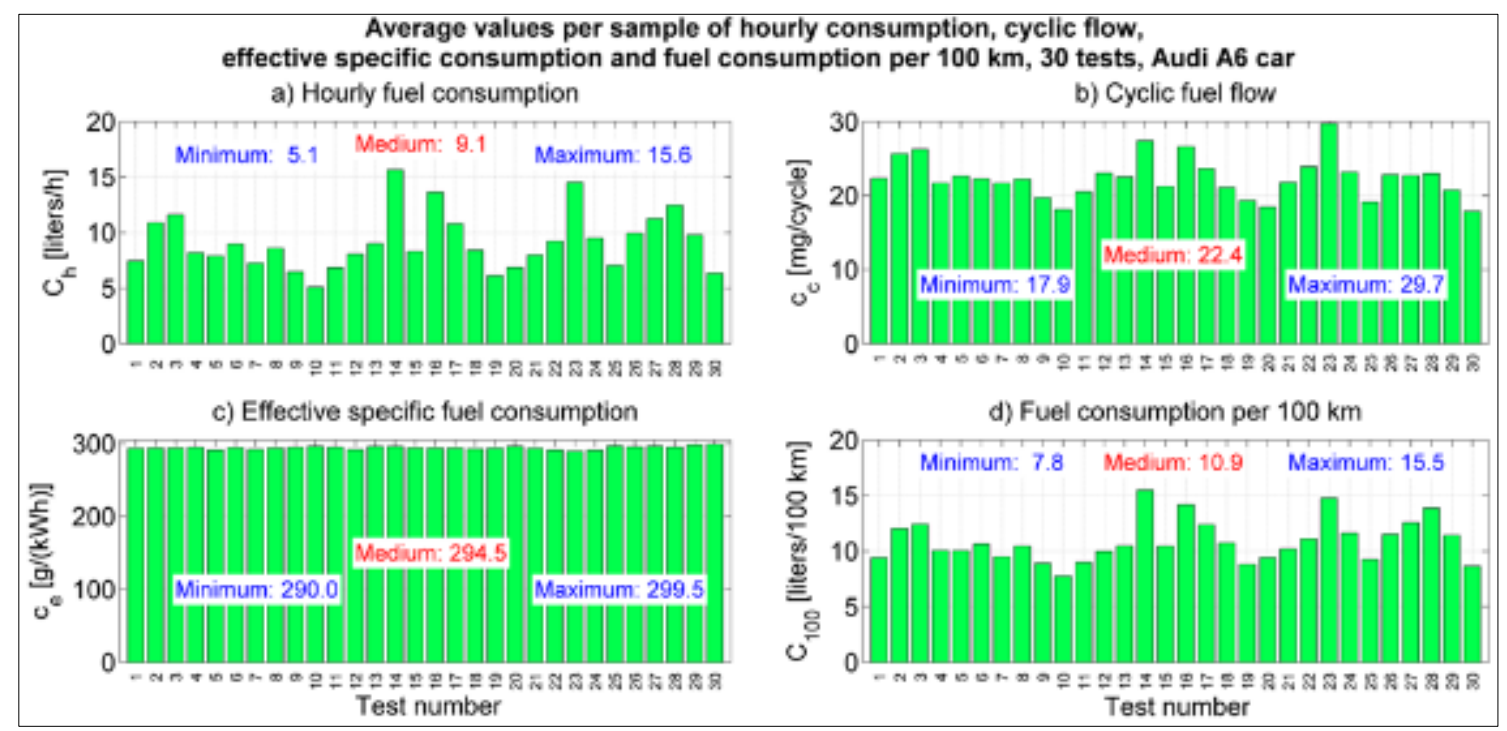

Figure 4 Mean values per test for four functional variables, 30 tests, Audi A6

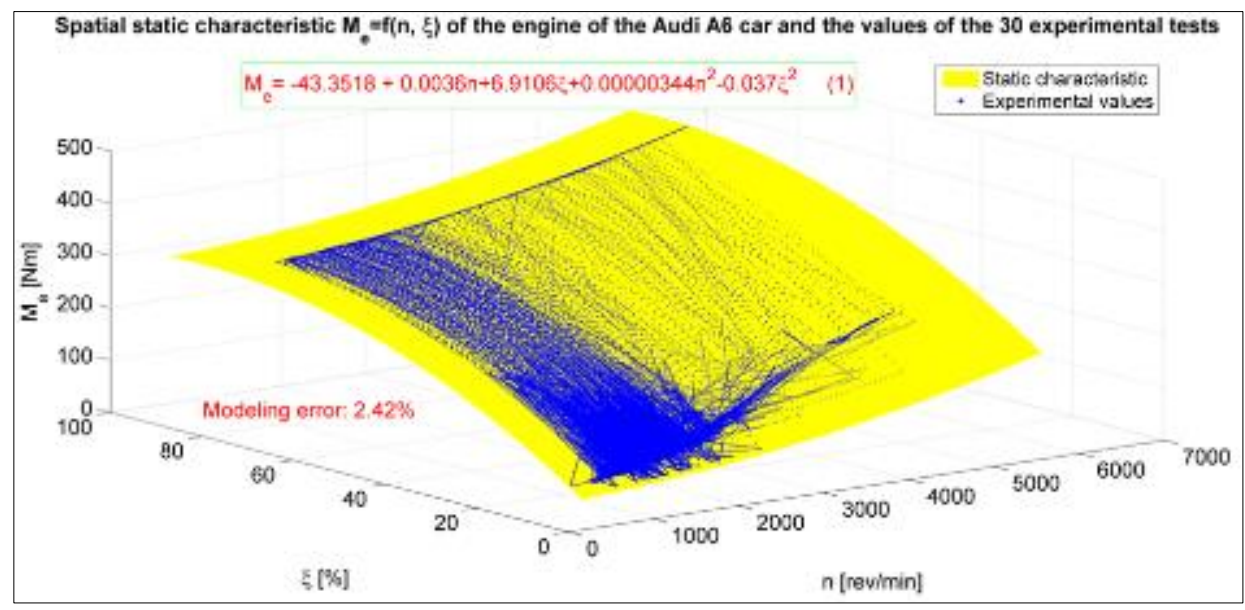

Figure 5 Spatial static characteristic $m e=f(n, \xi))$, Audi A6 car engine and 30 tests values 


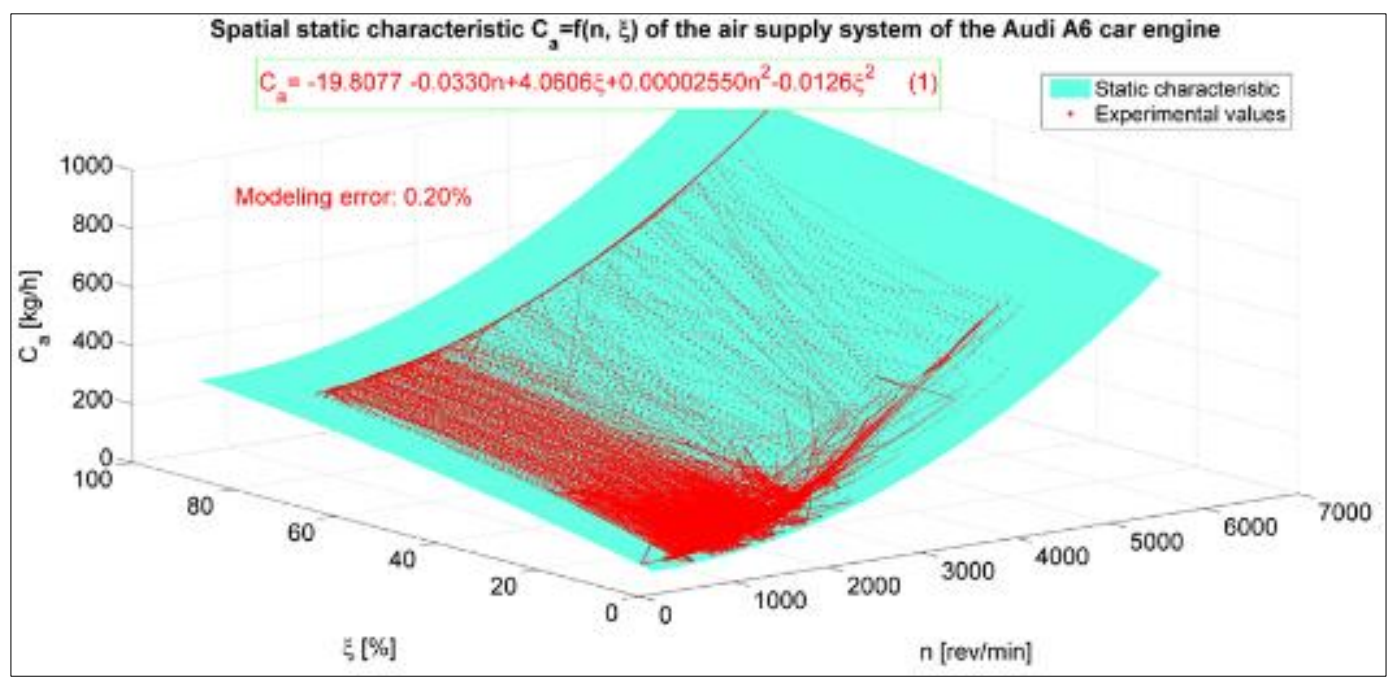

Figure 6 Spatial static characteristic $\left.C_{a}=\mathrm{f}(n, \xi)\right)$, Audi A6 car engine and 30 tests values

Fig. 5 shows the analytical expression (1) of the static characteristic $\left.M_{e}=\mathrm{f}(n, \xi)\right)$ :

$$
M_{e}(n, \xi)=-43.3518+0.0036 n+6.9106 \xi+0.00000344 n^{2}-0.037 \xi^{2}
$$

With $n$ engine speed and $\xi$ throttle position, all 3 variables being measured.

Fig. 6 also shows the analytical expression (1) of the spatial static characteristic $C_{a}=\mathrm{f}(n, \xi)$.

Fig. 7 shows the result of establishing the static characteristic $\lambda=\mathrm{f}(n, \xi)$ of the oxygen sensor of the Audi A6 car engine, where $\lambda$ represents the coefficient of air excess. The graph shows the switching surface of the static characteristic, its analytical expression (1), as well as the values of the 30 experimental tests.

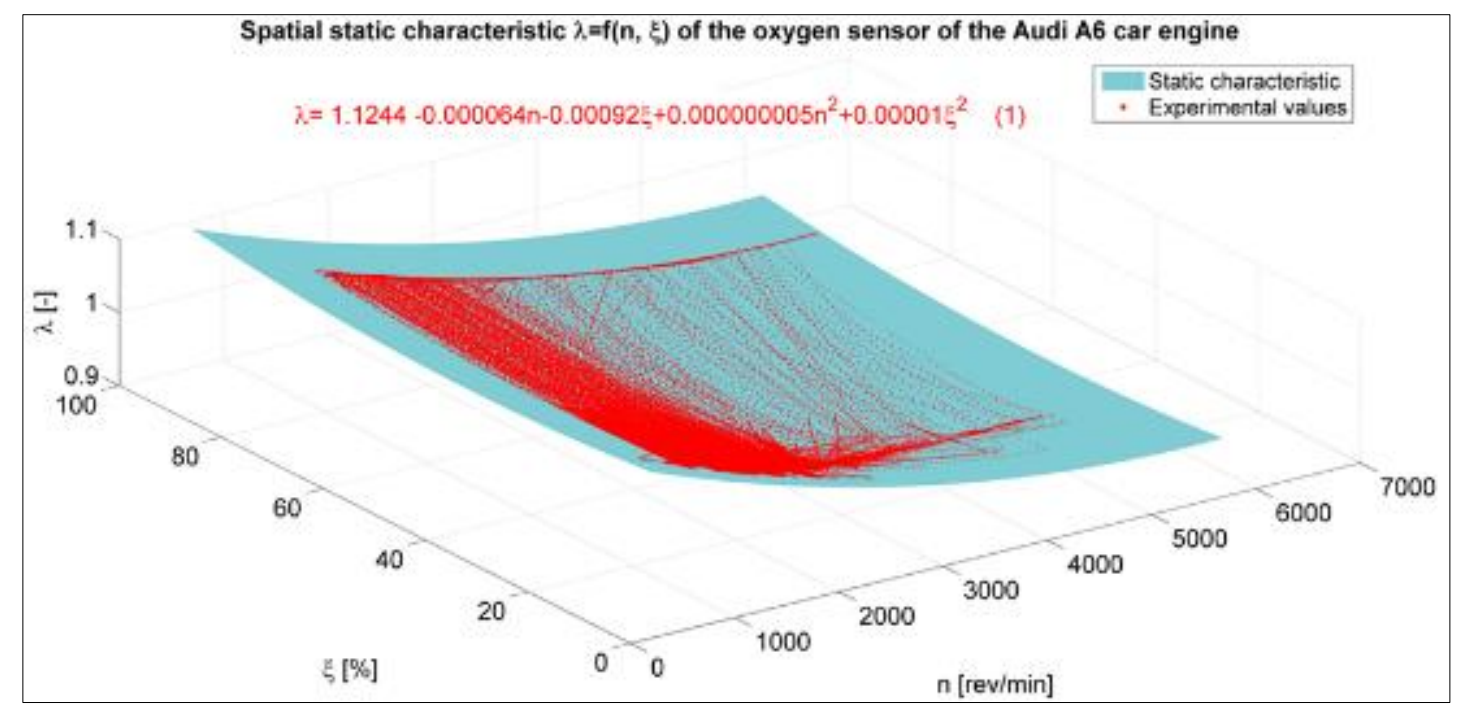

Figure 7 Spatial static characteristic $\lambda=\mathrm{f}(n, \xi)$, Audi A6 car engine and 30 tests values

Similarly, other static characteristics can be established when the engine is running without faults, which are used for comparison with the existence of faults. 


\section{Engine operation in the presence of several faults}

In this case, some faults were caused during the experiments; for a fault, 30 experimental tests marked D1-D30 were performed.

In this respect, the definition of a fault according to the SAFEPROCESS technical committee should be recalled. Thus, an impermissible deviation of at least one characteristic property / variable of the system from the acceptable / usual / standard / nominal behavior is called a fault; as can be seen from this definition, a fault means a deviation from the nominal value of a parameter or a certain functional variable.

In addition, the error is the quantitative measure of a fault and constitutes a deviation of the system parameters from their nominal values, or a deviation of a variable from its usual value (corresponding to a normal operation).

Finally, a failure means a fault that involves the permanent interruption of the system's ability to perform a required function under specified operating conditions; the failure can therefore be considered as a total fault (100\%).

Fig. 8 shows the case without fault (graphs on the left) and the case of a 75\% fault of the pressure sensor in the intake manifold (graphs on the right); by definition, the sensor had a measurement error of 75\%. The upper graphs show the time variations of the target variable, and the lower graphs show the time-frequency analysis by using the short-time Fourier transform.

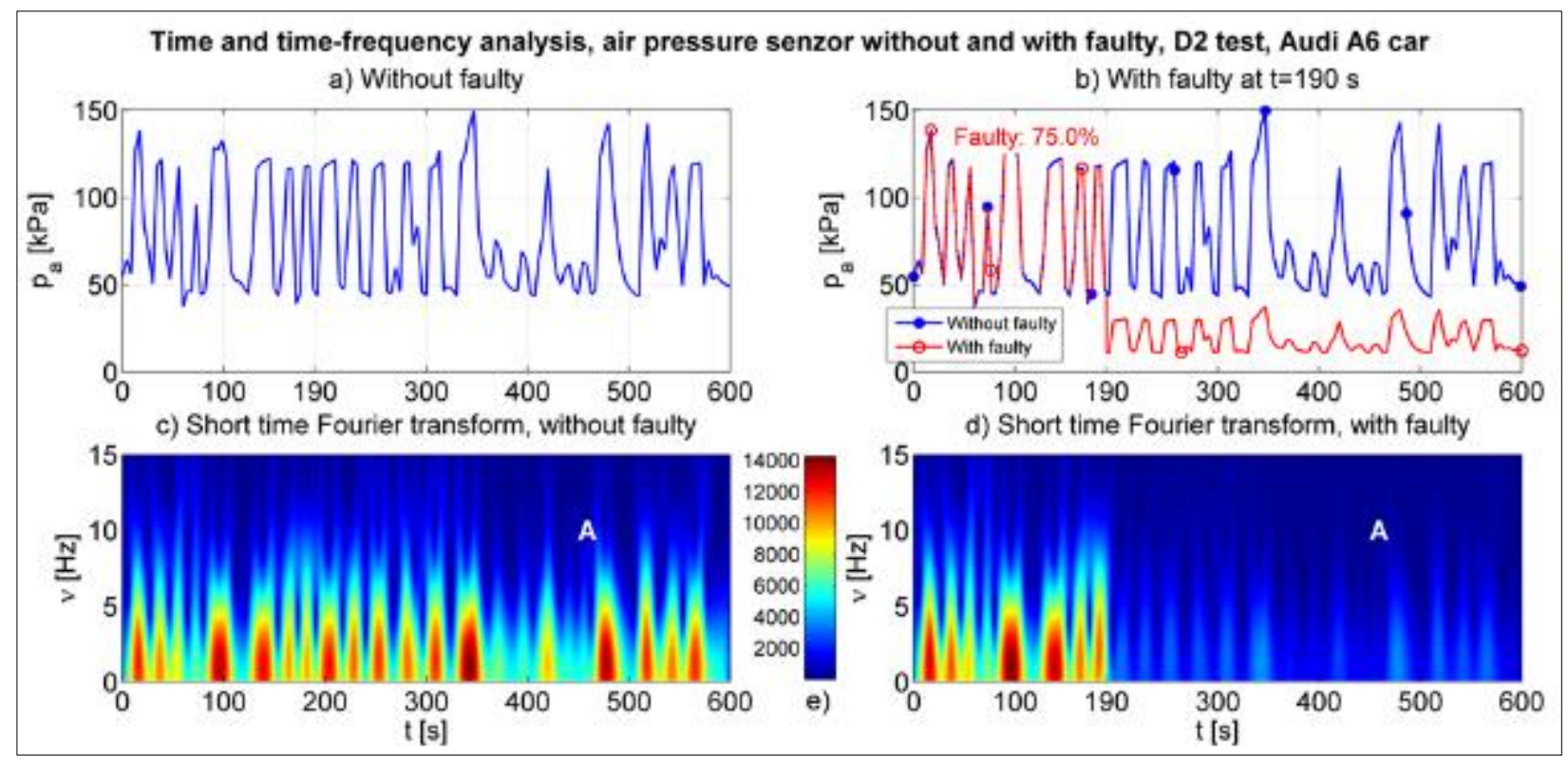

Figure 8 Time and time-frequency analysis, fault air pressure transducer, Audi A6

From fig. 8 it is found that the fault appeared at time $t=190 \mathrm{~s}$, which is observed both in the time domain in fig. $8 \mathrm{~b}$ and in the time-frequency analysis in fig.8d where the amplitude of the short-term Fourier transform (the amplitude with values in fig.8e) has lower values in zone A.

The short-time Fourier transform (STFT) of any variable $y$ is defined by the expression [3]:

$Y(t, j v, h)=\int_{-\infty}^{\infty} y(u) h^{*}(u-t) \mathrm{e}^{-j 2 \pi v u} \mathrm{~d} u$

where $h(t)$ represents the analysis window, $h^{*}$ its conjugate, $v$ is the frequency, $t$ and $u$ time.

It should also be noted that the spectrogram represents the square of the amplitude of the short-time Fourier transform: 
$S P(t, v)=\left|\int_{-\infty}^{\infty} y(u) h^{*}(u-t) \mathrm{e}^{-j 2 \pi v u} \mathrm{~d} u\right|^{2}$

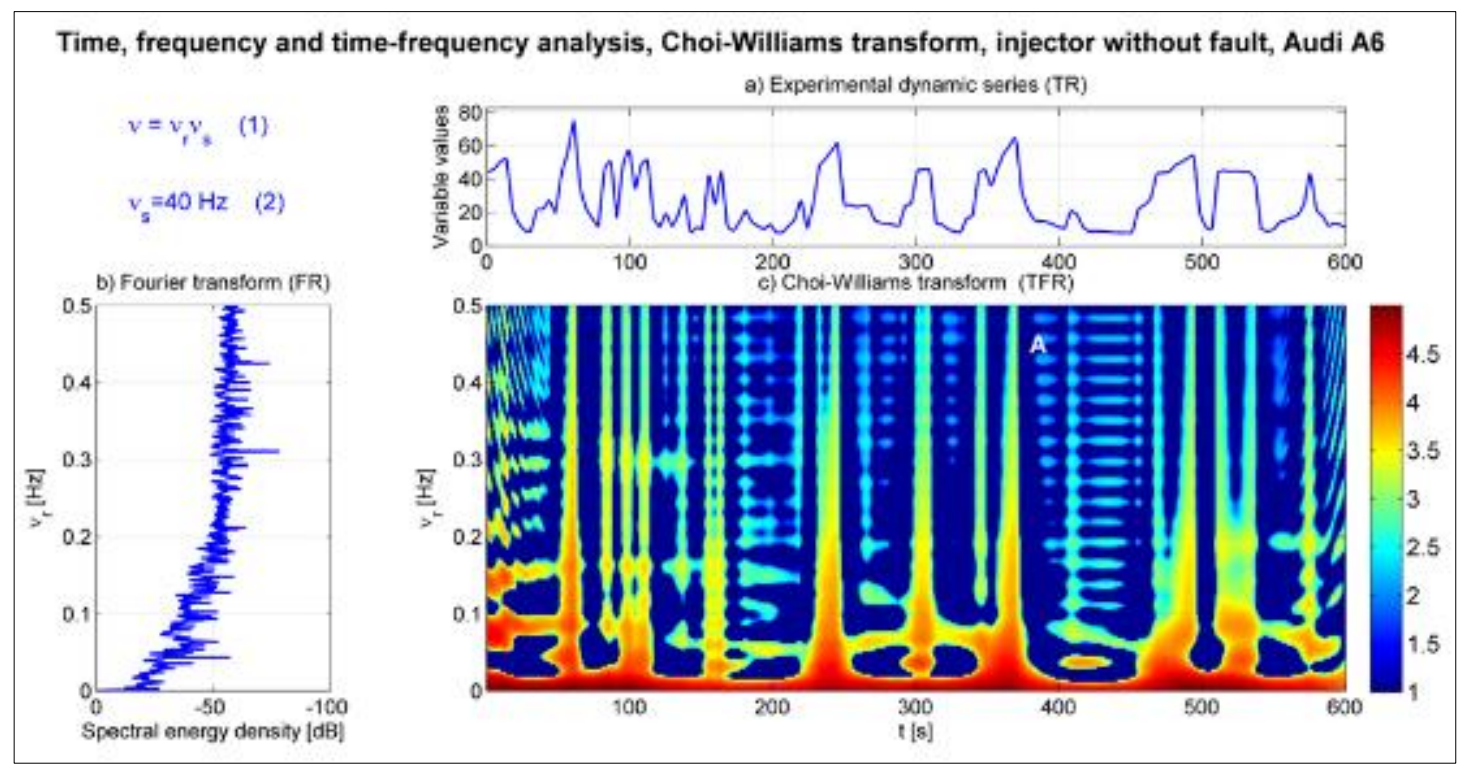

Figure 9 Time, frequency and time-frequency analysis, no injector fault, Audi A6

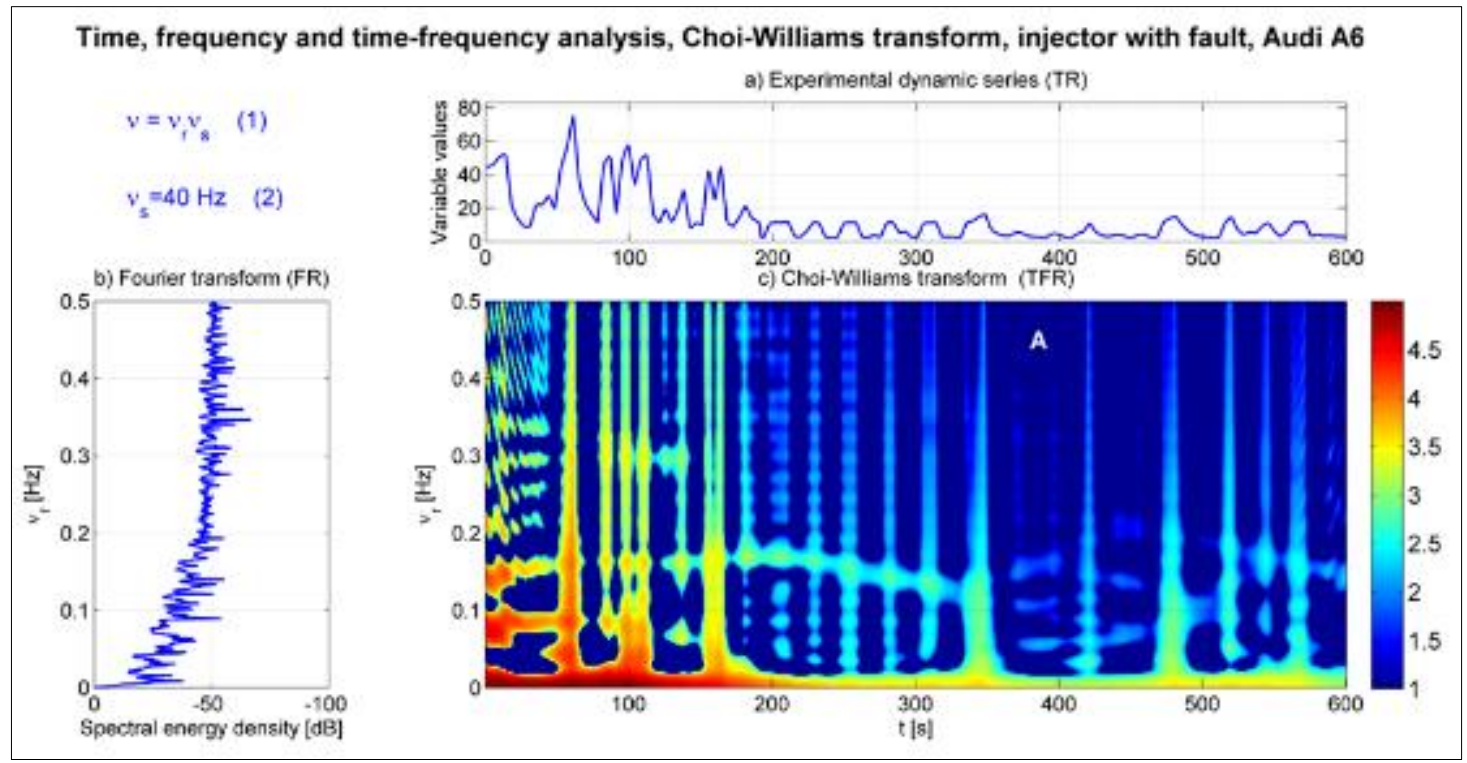

Figure 10 Time, frequency and time-frequency analysis, with injector fault, Audi A6

Fig. 9 and fig.10 aim at the occurrence of a fault in the injector, so the variable considered is the cyclic flow of fuel; in fig. 9 without defect, and in fig.10 with defect. In addition, all three types of analysis are used: time, frequency (by applying the classical Fourier transform) and time-frequency (by applying the Choi-Williams transform). As can be seen from fig.10, only the time representation (RT) and the time-frequency representation (RTF) detect the appearance of the fault, in the latter case by changing the image in area A (fig.10c) where the amplitude of the Choi-Williams transform decreases; the frequency representation (FR) does not detect the fault.

The Choi-Williams transform is defined by the relation, for some complex variable $y$ with his conjugate $y^{*}$ [5]: 
$Y(\tau, j u)=\sqrt{\frac{2}{\pi}} \int_{-\infty}^{\infty} \int_{-\infty}^{\infty} \frac{\sigma}{|\tau|} \mathrm{e}^{-2 \sigma^{2}(s-t)^{2} / \tau^{2}} y\left(s+\frac{\tau}{2}\right) y^{*}\left(s-\frac{\tau}{2}\right) \mathrm{e}^{-j 2 \pi v t} \mathrm{~d} s \mathrm{~d} \tau(4)$

Where $t, \tau$ and $s$ are time, and $\sigma$ the standard deviation of the variable $y$.

In the graphs in fig.9c and 10c, on the ordinate axis was represented the normalized frequency, or the relative frequency $v_{r}$ (Nyquist frequency being $0.5 \mathrm{~Hz}$ ); the time $t$ is rendered on the abscissa axis. The absolute frequency is obtained by multiplying the number on the ordinate axis in fig.9c and 10c with the sampling frequency $v_{s}$ according to relation (1); the axis of the ordinates in fig.9c and fig.10c is the same as the axis of the abscissas in fig. $9 \mathrm{~b}$ and fig.10b, where the graph is arranged rotated to the left by $90^{\circ}$.

Fig. 11 shows the analysis in time and time-frequency of the signal from the oxygen sensor, the functional variable being the coefficient of excess air $\lambda$. For the time-frequency analysis we used the $S$ transform (defined by Stockwell), which is an extension of the wavelet transform through a phase correction of it and which is defined by the relation [1,5]:

$Y(\tau, j v)=\int_{-\infty}^{\infty} y(t) \frac{|v|}{\sqrt{2 \pi}} \mathrm{e}^{-\frac{(t-\tau)^{2} v^{2}}{2}} \mathrm{e}^{-j 2 \pi v t} \mathrm{~d} t$

where in the expression marking including the wavelet extension is:

$\varphi(\tau, v)=\frac{|v|}{\sqrt{2 \pi}} \mathrm{e}^{-\frac{(t-\tau)^{2} v^{2}}{2}} \mathrm{e}^{-j 2 \pi v t}$

Fig. 11 highlights the areas B and D in which the fault occurred in the oxygen sensor, in this case in fact a failure. The graduated scale on the right in Fig. 11b highlights the values of the amplitude of the Stockwell transform.

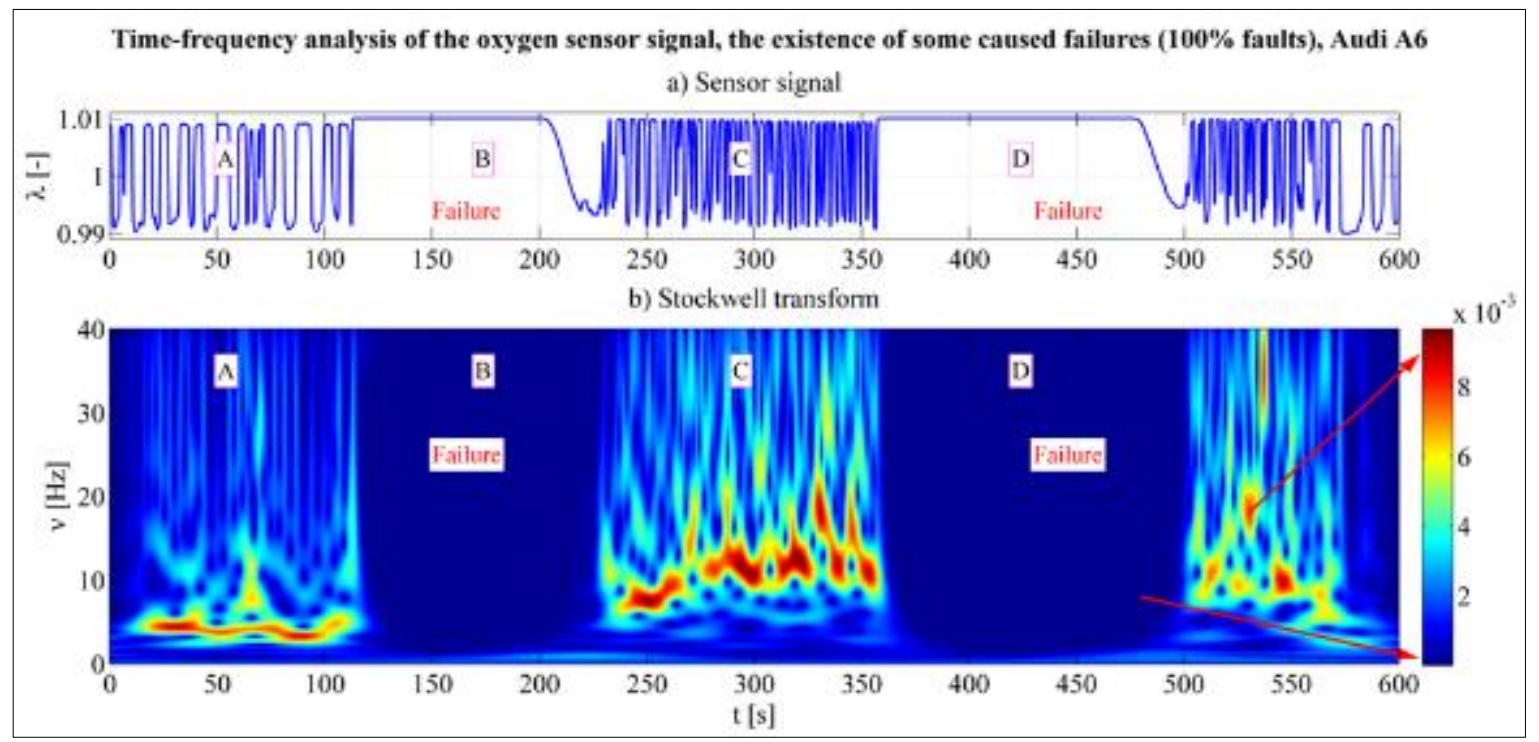

Figure 11 Time and time-frequency analysis, oxygen sensor, Audi A6 car

Fig.12 and fig.13 show the analysis in time and time-frequency in the case without fault (fig.12) and in the situation with fault (fig.13) at the injector; the graphs in fig.12c and fig.13c highlight different pictures in zone A. For the timefrequency analysis, the scalogram was used, which represents the square of the amplitude of the wavelet transform [4] and which is defined by the expression (with the scale parameter $a$ ): 


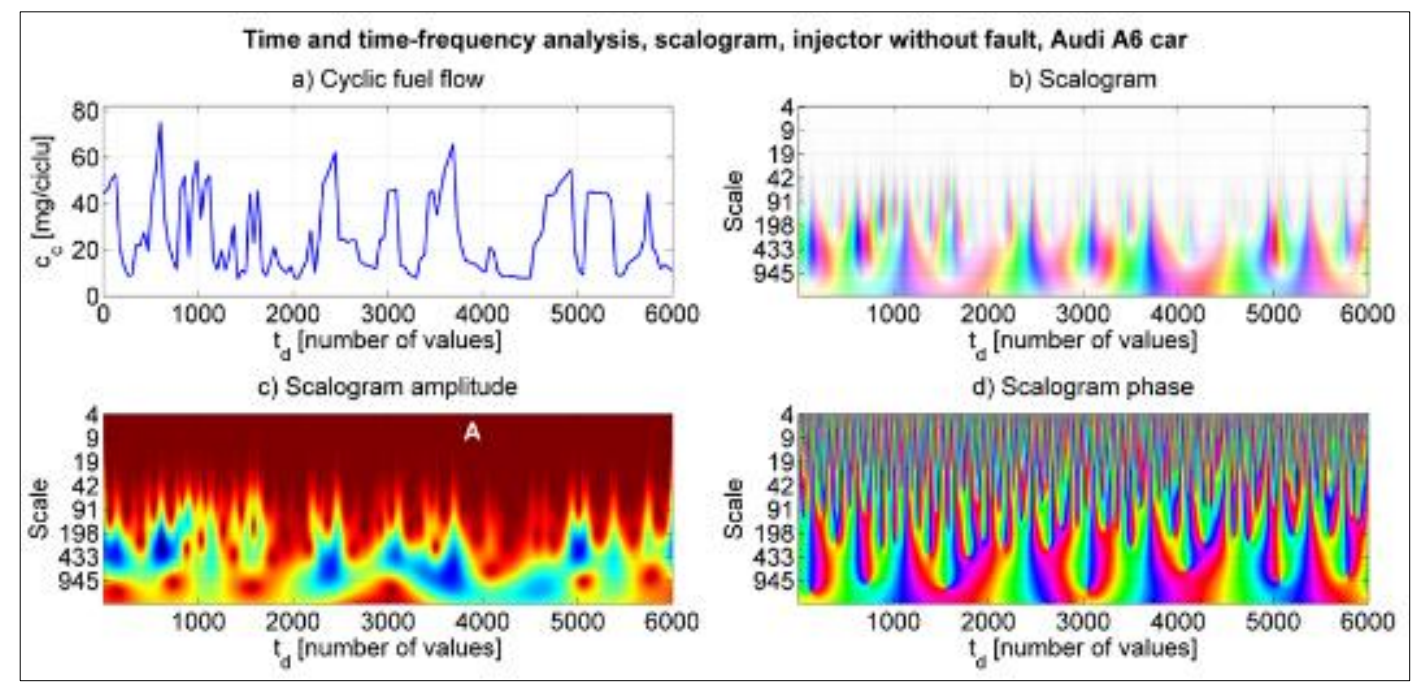

Figure 12 Time and frequency analysis, fault-free injector, Audi A6 car

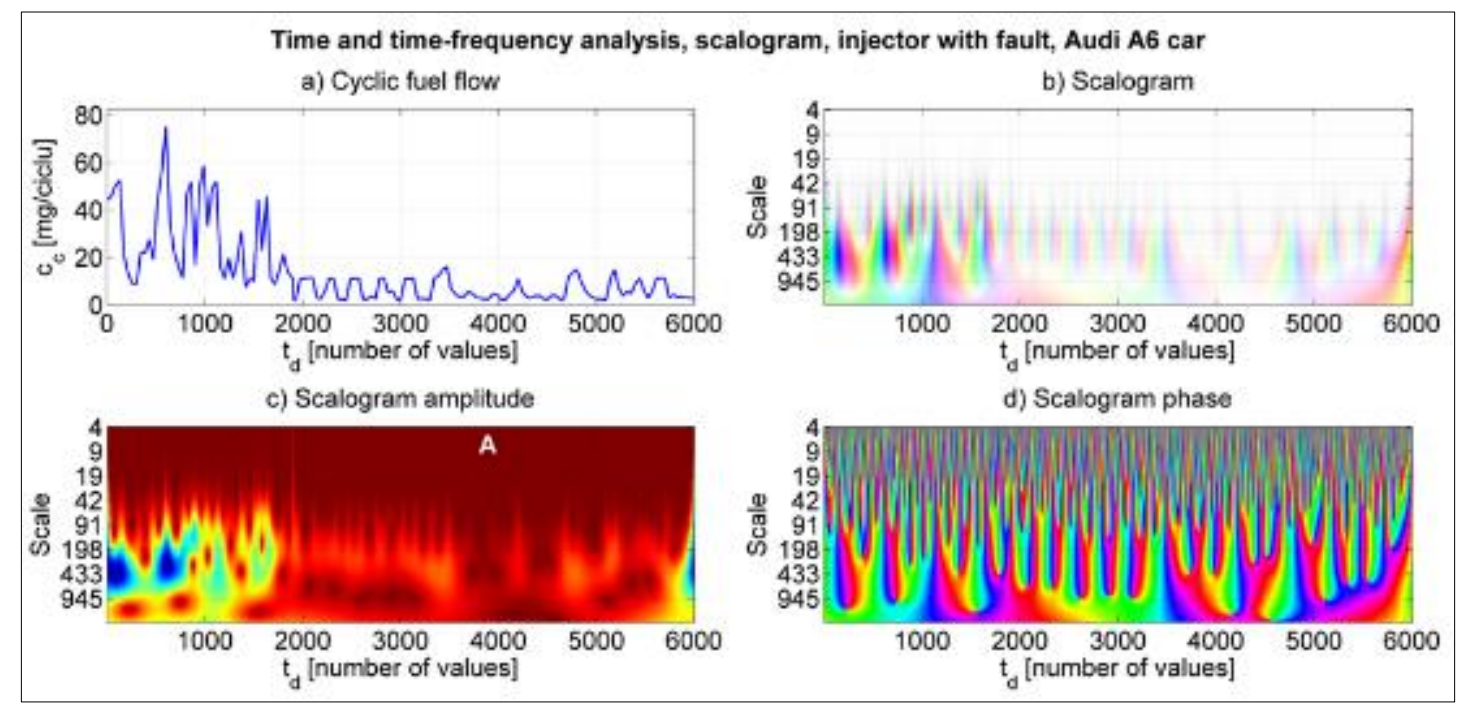

Figure 13 Time and time-frequency analysis, injector with fault, Audi A6 car

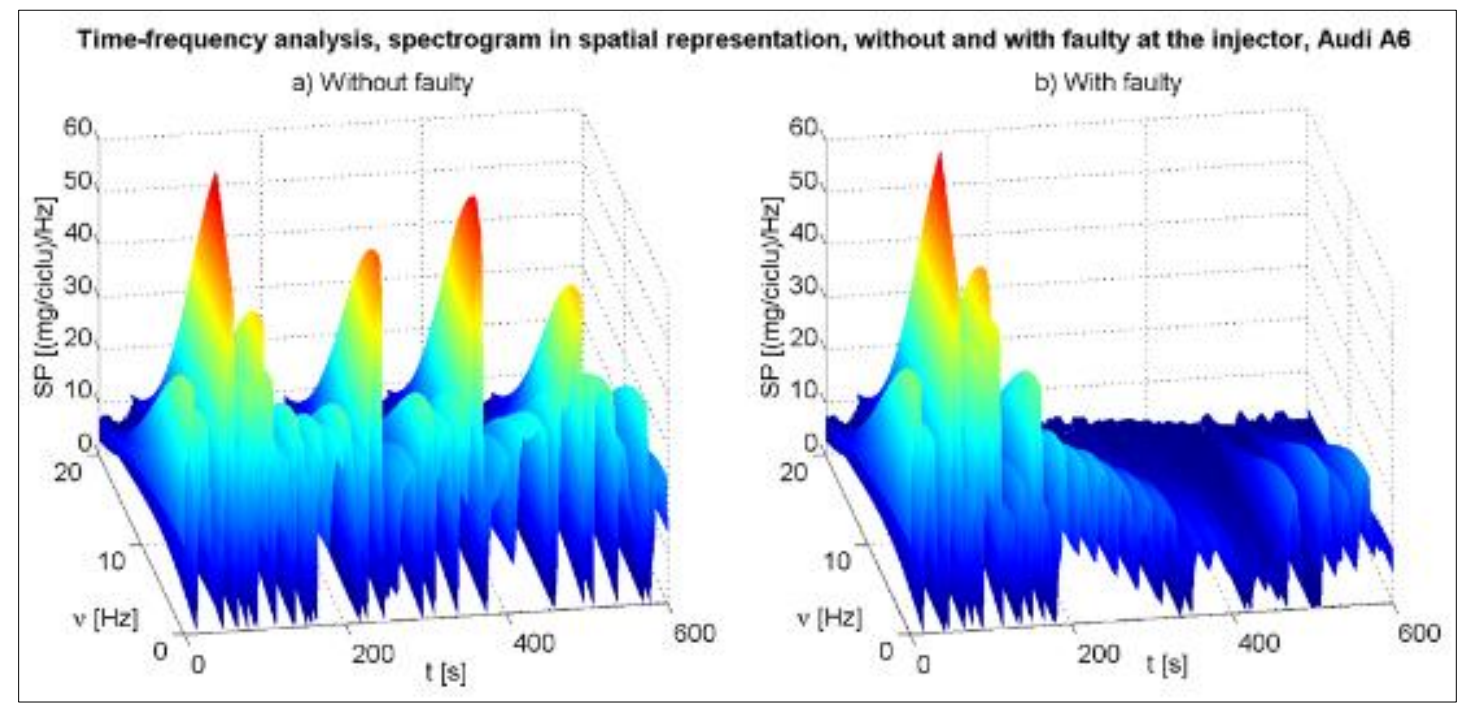

Figure 14 Time-frequency analysis, spatial spectrogram, injector, Audi A6 car 


$$
S C(t, a ; h)=\left|\frac{1}{a} \int_{-\infty}^{\infty} y(s) h^{*} \frac{s-t}{a} \mathrm{~d} s\right|^{2}
$$

The time-frequency analysis can also benefit from a spatial representation, as it is found in the spectrograms from fig.14, in fig. $14 \mathrm{~b}$ being noticed the decrease of the spectrogram amplitude.

\section{Conclusion}

The diagnosis of gasoline injection engines currently uses new methods and algorithms in addition to the classic onboard diagnostic solution. Among these methods is signal-based diagnostics, which benefit from built-in sensors and actuators and new signal processing algorithms. When signal-based diagnostics, time-frequency analysis techniques are used a lot, which specify the time when there is a fault and its size.

\section{Compliance with ethical standards}

\section{Disclosure of conflict of interest}

No conflict of interest to declare.

\section{References}

[1] Jianhua C, Yongliang X. Time-frequency analysis method of bearing fault diagnosis based on the generalized S transformation. JVE International ltd. journal of vibroengineering. SEP 2017; 19(6).

[2] Giantomassi A, Ferracuti F, Iarlori S, Ippoliti G, Longhi S. Signal Based Fault Detection and Diagnosis for rotating electrical machines: Issues and Solutions.

[3] Schiiler M, Leonhardt S, Ludwig C, Ayoubi M, Isermann R. Model-based and signal-based fault detection of diesel engines with turbocharger.

[4] Vong CM, Wong PK. Engine ignition signal diagnosis with Wavelet Packet Transform and Multi-class Least Squares Support Vector Machines.

[5] *** The Time-Frequency Toolbox (tftb) for Matlab.

[6] Barothi L., Stoica R.-M., Voicu D., Singureanu M. Simulation and comparative analysis of pollutant emissions before and after PID control of engine functioning. Ingineria Automobilului, nr. 56. sep. 2020; 19-23.

[7] Vilǎu R, Lespezeanu I, Singureanu M. Considerations regarding the influence of inappropriate electronic throttle unit operation on pollutant emissions of internal combustion engines. 5th International Scientific Conference SEA-CONF 2019, IOP Conf. Series: Journal of Physics: Conf. Series. 2019; 1297: 012032. 